\title{
General Discussion to papers of J. B. Cook et al., H. J. Hachen and G. Ravichandran
}

Mr E. GRIffiths Chairman. Perhaps the three authors could be near a microphone to answer questions.

SIR LUDWIG GUTTMANN (G.B.). Dr Ravichandran made a question mark behind your title. I think you answered the question that it is a progressive disease all right. The only problem is why is it that a very extensive haemangioma starting in the cervical and the paraplegia is down to $\mathrm{T}_{4}$ ? I can give you the answer because I have described such a case, you will find it in my book. The reason is in a certain area the convolution, the venous convolution become thrombosed and act as a tumour, in other words as a compression on that area of the spinal cord and that is a possibility where perhaps surgical intervention can help.

I have been particularly interested in the paper of Burt and Cook on acute spinal epidural abscess. Now this is a very important process which I think is often wrongly diagnosed. Dr Burt already mentioned the wrong diagnosis in his case. I would like to add in particular, patients who develop paraplegia and are transferred to a general medical department where the diagnosis of transverse myelitis is made, but after transfer to our Spinal Centre, turn out to be epidural abscesses. We had, I think, four or five such patients. Actually, one was my very first patient. He was a traumatic lesion below LI and developed a swelling on his left buttock. I aspirated a huge abscess without opening up which fortunately healed and in due course the patient was discharged following rehabilitation and has been working for many years. Some of the other cases came at night, and once the diagnosis of epidural abscess was made in view of the natural history they were operated by me at once and the abscess verified and treated accordingly. Dr Burt mentioned quite rightly that that man had before he developed the pain some infection in the same area and that is a point we have to consider as a very important indication for epidural abscess. People may develop a little boil or abscess in other parts of the body then they start to complain about girdle pain and local tenderness in the thoracic area. This may be mis-diagnosed if there is no rise of temperature as a disc or low back pain, as it happened in one of our cases, of any other cause. There are two types of epidural abscess, one is the mono-loculated abscess, the other type when you open up the skin and the fascia there you will find pus coming out from various parts of the exposed muscle area. Unfortunately the people come too late, because once the infection has affected the vascular supply, particularly the vein, then you get ischaemia of the spinal cord and there is no recovery whatsoever. Therefore, in all cases of suspected epidural abscess the most important thing to do is to transfer the patient before you make the diagnosis of tranverse myelitis to the nearest spinal unit or neuro-surgical unit. As mentioned, in one case we failed; that was a man with $\mathrm{T}_{4}$ lesion who came to us and who already had disc trouble before. I wasn't quite certain about this and I felt we had better transfer him to Pennybacker at Oxford the expert on discs. They opened up, it was an abscess.

Mr GRIfFiths. Can I just make a comment myself about this and then we'll take the question? May I ask Dr Hakin? We've had of course the embolic epidural abscesses, two or three of these, which of course need surgery and they should be picked up early. We are not confusing this with spinal osteomyelitis of the vertebral bodies, of which we've had perhaps 12 in the last 2 years, but with X-ray changes, and frankly abscesses cause compression. All these I presume were those of embolic sepsis with no bony changes. Is that so ?

DR HAKIN (G.B.). I think I might make one or two points about that. I'm not sure that you can divide them into embolic straight into the abscess cavity as it were and embolic to the bone. My impression is that these people complain of excruciating back pain with tenderness and I've spoken to one or two neuro-surgeons about this and their feeling is that the pain is due to an osteitis, in other words, it isn't just that the 
infection gets into the extradural space. The feeling is in those I discussed it with, is that the infection is actually there within shall we say the lamina or the spine and then goes into the extradural space and I don't believe that you can divide them up like that. I know that there have been various papers, for instance Hoisner wrote a paper in the late I940s in which he subdivided them into the so-called metastatic and the so-called osteomyelitic and he thought that there was some difference in the age distribution. My only question is how does it get into the extradural abscess as it were and how does it get into the bone, they must both be metastatic I would have thought. I think there must be an osteomyelitic process whatever.

MR GRIFFITHS. I just wonder whether X-ray changes on the plain X-rays of the other cases quoted.

DR HAKIN. They were all negative and even with the retrospectoscope, we've asked two or three neuro-radiologists and they said they could see no alteration.

SIR LUDWIG GUTTMANN. With regard to the osteomyelitic type of epidural abscess the plain X-ray might now show the osteomyelitic changes. I remember two cases, one in particular, a naval officer with a mid-thoracic paraplegia following bomb injury and continuous sinus and X-ray didn't show anything. But when I injected lipiodol into the sinus the sinus penetrated into the bone. I scraped it out and it healed for some time but, unfortunately, it recurred again. Once the osteomyelitis in the vertebral body is diagnosed it may take a very long time until it heals, if at all.

Dr Chawla (G.B.). I'd like to ask Dr Ravichandran a question about the possible aetiology of the cause of the symptoms and signs in these patients, because apart from the ischaemia with regard to venous shunt and local pressure as Sir Ludwig has mentioned of large haemangioma a large blood vessel malformation, there is also an arachnoiditis in these patients and this is due to minor bleeds which these patients tend to suffer. One of the symptoms of arachnoiditis is burning pain. Did any of these patients have this burning pain?

Dr G. RavichandRan (G.B). Three of the I3 patients had burning pain. The majority of the patients had weakness as a primary symptom and radicular pain as the next close symptom.

DR K. R. KRISHNAN (G.B.). I want to go back to Dr Hachen's paper, and I am pleased that he ended his excellent paper with a plea for mitigation. Because if we can look at an average casualty department, the people who come with back pain and tenderness of the spine and whatever, it must be a pretty difficult job for a casualty officer to understand the implications. In our experience in Southport we have had two cases of epidural abscess, fortunately both of them made a reasonable recovery and are walking with aids. So the one point on which I wasn't clear. He mentioned about PUO in one of his I 2 patients. I would have thought it would have been an interesting dictum to remember. If you get a patient with some form of neurological lesion attributable to the spine, with a pyrexia, I feel it is absolutely essential to think that unless otherwise proved this man most probably has an epidural abscess. So pyrexia and paresis, I think it probably the most important diagnostic combination for an epidural abscess. The second point I want to make again in mitigation is that a large number of these patients shown as NAD in a little casualty card by the casualty officer, or by the orthopaedic registrar, is very rarely NAD and it doesn't really give us any information whatsoever, because neurological examination by a neurological registrar or whatever will soon know NADs are not really NADs at all.

Mr Griffiths. I agree entirely with you. A final I think from Jack Walsh, please?

DR J. WALSH (G.B.). Just one question to Dr Hakin with regard to the epidural abscess. The two cases I saw here in the first few hours had one outstanding sign. They had acute, very localised and very acute tenderness over the area of the abscess proved eventually. I wonder how many of his cases had the same sign?

DR R. N. HAKIN. Eleven out of I2 and specifically this last gentleman on each occasion that he went to the Casualty Department they stated in the notes quite categorically that there is exquisite tenderness D8, 9, IO. Three occasions, that's what they wrote in the notes. 
Can I just come back to one previous point? This business about a focus of infection which presents days or weeks or even in some cases as long as 3 months prior to the onset of the neurological symptoms from the abscess. It is very nice if they have a focus of infection prior to the epidural abscess, but I am afraid it doesn't happen in more than 50 per cent of cases and that makes it even more difficult I'm afraid.

DR F. W. MEINECKe (Germany). I'd like to come back to Sir Ludwig's comment because you raise a very important point there that the relation of any minor or severe trauma with the abscess and that is very important for the liability insurances in particular for the Workman's compensation part. Do you feel that infection may be a very superficial local skin infection? It must be or should be causal for the abscess and to come to the next step, if you find an abscess and the man has had a minor trauma do you feel that this is doubtless the cause of the abscess or may there be some other reason for that because the solicitors will be asked to answer this question very carefully, the history of injury preceding the infection?

DR R. N. HAKIN (G.B.). Yes, I read all the papers and they say in about a third with a preceding history of trauma. I must say it has always seemed to me a rather vague story. If you actually went round this audience and asked how many of them had had a bit of a bang on the back in the last few months I would think you could probably find somewhere between 20 and 30 per cent might very well say yes. I'm not convinced that there is actually a haematoma which forms a nidus for the embolic infection. It may be so but I must say I am not altogether convinced.

MR E. R. Griffiths. Thank you very much, and I must say I think we should thank the last three authors for a very good combination of papers. 